\title{
THE MULTIPLICITY OF ISOLATED TWO-DIMENSIONAL HYPERSURFACE SINGULARITIES
}

\author{
HENRY B. LAUFER
}

\begin{abstract}
Consider an isolated two-dimensional complex analytic hypersurface singularity $(V, p)$. A relation is given between the abstract topology of $(V, p)$ and the multiplicity of $(V, p)$, yielding an upper bound for the multiplicity. This relation is a necessary condition for a Gorenstein singularity to be a hypersurface.
\end{abstract}

I. Introduction. Let $(V, p)$ be an isolated two-dimensional hypersurface complexanalytic singularity. In this paper we ask: What conditions are imposed on the abstract topology of $(V, p)$ by the hypersurface hypothesis? Recall, essentially [6, Theorem 2.10, p. 18], that any isolated singularity is a cone over its link $L$. Moreover, in dimension two, $L$ is a compact real 3-manifold whose oriented homeomorphism type determines and is determined by the weighted dual graph $\Gamma$ of a canonically determined resolution $\pi:(M, A) \rightarrow(V, p)$ [7]. So, we may equivalently ask: What conditions does the existence of a hypersurface representative $(V, p)$ put on a weighted dual graph $\Gamma$ ? A hypersurface singularity $(V, p)$ is Gorenstein [2, 4]. So there exists an integral cycle $K$ on $\Gamma$ which satisfies the adjunction formula (2.1) below [9]. This paper is primarily devoted to deriving an additional necessary relation, Theorem 3.1, between $K$ and the pull-back to $(M, A)$ of the maximal ideal $\mathrm{m}$ of the hypersurface $V$ at $p$. This relation is in general not satisfied by $(V, p)$ which are merely Gorenstein. Consider Example 4.1, the simple elliptic singularities $[8,5]$. These are Gorenstein for all values of $Z \cdot Z$, but are hypersurfaces only for $-Z \cdot Z \leqslant 3$. These singularities fail to satisfy the condition of Theorem 3.1 for $-Z \cdot Z>3$.

This paper was somewhat inspired by the Zariski multiplicity question [12]. Some very mild implications towards a positive answer to Zariski's question do follow from Theorem 3.1. An upper bound, Corollary 3.6, is obtained for the multiplicity of a hypersurface $(V, p)$ in terms of $\Gamma$ of the minimal resolution. A lower bound is well known to be given by $-Z \cdot Z$, where $Z$ is the fundamental cycle $[\mathbf{1}, \mathbf{1 0}]$. Unfortunately, often neither bound is sharp. One cannot hope always to determine the multiplicity of $(V, p)$ from its nonembedded topology alone, or even from the topology and the Milnor number. Namely, Example 4.3 includes two surface

Received by the editors September 3, 1985 and, in revised form, June 5, 1986.

1980 Mathematics Subject Classification (1985 Revision). Primary 32C40, 32C45, 14B05; Secondary $32 \mathrm{~B} 30$. 
singularities which have different multiplicities but the same $\Gamma$ and the same Milnor numbers. These two singularities do have very different characteristic polynomials [6].

II. Known preliminaries. Consider a resolution $\pi:(M, A) \rightarrow(V, p)$ of the normal two-dimensional singularity $(V, p)$. Throughout this paper $A=\bigcup A_{i}, 1 \leqslant i \leqslant n$, will be the decomposition of $A$ into irreducible components. Consider the canonical bundle $K$ on $M$. The adjunction formula [9] gives, for all $i$,

$$
A_{i} \cdot K=-A_{i} \cdot A_{i}+2 g_{i}-2 .
$$

Recall [3], that an $A_{j}$ is an exceptional curve of the first kind, i.e. may be blown down without introducing a singularity, if and only if $A_{j}$ is a nonsingular rational curve of self-intersection -1 . Observe then from (2.1) that

$$
\begin{aligned}
& A_{i} \cdot K=-1 \text { if } A_{i} \text { is exceptional of the first kind, } \\
& A_{i} \cdot K \geqslant 0 \text { otherwise. }
\end{aligned}
$$

$\pi$ is the minimal resolution if and only if no $A_{i}$ is exceptional of the first kind.

Since the intersection matrix $\left(A_{i} \cdot A_{j}\right)$ is nonsingular, there are unique rational numbers $k_{i}, 1 \leqslant i \leqslant n$, such that the rational cycle

$$
\tilde{K}=\sum k_{i} A_{i}, \quad 1 \leqslant i \leqslant n
$$

satisfies $A_{i} \cdot K=A_{i} \cdot \tilde{K}$ for all $i$.

Proposition 2.1 is certainly known, but seems not to have appeared in the literature.

Proposition 2.1. With the above notation, suppose additionally that $\pi$ is the minimal resolution. Then $K=0$, i.e. is the trivial bundle, in case $(V, p)$ is a rational double point. Otherwise, $k_{i}<0$ for all $i$.

Proof. Rational double points are classified in [1]. So we may consider only the second case, $\tilde{K} \neq 0$, and assume $A_{j} \cdot K>0$ for some $j$. Using rational coefficients, write $\tilde{K}=C+D$, where $C \leqslant 0$ and $D \geqslant 0$. We assume that $D \neq 0$ and reach a contradiction: Since $D \neq 0, D \cdot D<0$. Then there exists $A_{j}$ such that $A_{j} \cdot D<0$. Since $C \leqslant 0, A_{j} \cdot C \leqslant 0$. Then $A_{j} \cdot K<0$, contradicting the hypothesis that $\pi$ is the minimal resolution. Hence $\tilde{K} \leqslant 0$. Since $\tilde{K} \neq 0$, should $k_{j}=0$ occur for some $j$, then we may further choose $j$ so that $A_{j} \cdot A_{l}>0$ for some $A_{l}$ with $k_{l}<0$. Then $A_{j} \cdot K<0$, which again contradicts the minimality of $\pi$.

Recall that for any Cartier divisor $D$ on $M, \mathcal{O}(D)$ denotes the sheaf of germs of meromorphic functions $f$ such that (locally), $(f)+D \geqslant 0$. We let $\mathcal{O}(K)$ denote the sheaf of germs of sections of the canonical bundle $K$. In case $(V, p)$ is Gorenstein, the $\tilde{K}$ of (2.2) has integral coefficients. Moreover, $\mathcal{O}(\tilde{K})$ is isomorphic to $\mathcal{O}(K)$. Thus in the Gorenstein case, we shall duplicate notation and use $K$ also to denote the Cartier divisor $\tilde{K}$.

From now on in this paper, all "cycles" will be integral combinations of the $A_{i}$, i.e. Cartier divisors on $M$. 
Let $\mathfrak{m}$ denote the maximal ideal sheaf at $p$ of $(V, p) . \pi^{*}(\mathfrak{m})$, the sheaf on $M$ generated by the pull-back to $M$ of generators of $\mathfrak{m}$, need not be locally principal. But there is a unique cycle $D>0$ such that $\pi^{*}(\mathrm{~m}) / \mathcal{O}(-D)$ is supported at only a finite number of points, called embedded points (for $\pi^{*}(\mathrm{~m})$ ), on $A$. Following Yau [11], we shall call $D$ the maximal ideal cycle. There is a minimal resolution $\pi^{\prime}$ : $\left(M^{\prime}, A^{\prime}\right) \rightarrow(V, p)$ with $\pi^{\prime *}(\mathfrak{m})$ locally principal, i.e., such that $\pi^{\prime *}(\mathrm{~m})$ has no embedded points. $\pi^{\prime}$ is obtained by starting with the minimal resolution $\pi$ and successively blowing up at all embedded points. Let $\nu$ denote the multiplicity of $V$ at $p$. Then [10] $\nu=-D^{\prime} \cdot D^{\prime}$. Consider any two resolutions $\pi^{\prime \prime}$ and $\pi^{\prime \prime \prime}$ of $(V, p)$ with corresponding maximal ideal cycles $D^{\prime \prime}$ and $D^{\prime \prime \prime}$. If $\pi^{\prime \prime \prime}$ is obtained from $\pi^{\prime \prime}$ by blowing up at an embedded point, then $-D^{\prime \prime \prime} \cdot D^{\prime \prime \prime} \geqslant 1-D^{\prime \prime} \cdot D^{\prime \prime}$. Hence at most $\nu-1$ blowups are required to reach $\pi^{\prime}$ from $\pi$. Those resolutions $\pi^{\prime \prime}:\left(M^{\prime \prime}, A^{\prime \prime}\right) \rightarrow$ ( $V, p$ ), including $\pi$ and $\pi^{\prime}$, which appear in going from $\pi$ to $\pi^{\prime}$ via blowups may be characterized by: if $A_{j}^{\prime \prime}$ is an exceptional curve of the first kind, then $A_{j}^{\prime \prime} \cdot D^{\prime \prime}<0$.

III. Main results. We continue with the notation of §II.

THEOREM 3.1. Let $(V, p)$ be an isolated two-dimensional hypersurface singularity with multiplicity $\nu$. Let $\pi:(M, A) \rightarrow(V, p)$ be a resolution. Let $D=\sum d_{i} A_{i}$ be the maximum ideal cycle. Suppose that for each $A_{j}$ which is exceptional of the first kind, $A_{j} \cdot D<0$. Then for all $A_{i}$,

$$
-k_{i} \geqslant 1+(\nu-3) d_{i} .
$$

Theorem 3.1 will be proved in stages.

Proposition 3.2. Let $\pi:(M, A) \rightarrow(V, p)$ be as in Theorem 3.1. Consider an $A_{i}$ in $A$.

(i) If $A_{i} \cdot D<0$ and $\pi^{*}(\mathrm{~m})$ has no embedded points on $A_{i}$, then there exist elements $x$ and $y$ of $\mathrm{m}$ such that $\pi^{*}(x)$ and $\pi^{*}(y)$ restrict to linearly independent (over C) elements of $\Gamma\left(A_{i}, \mathcal{O}(-D) / \mathcal{O}\left(-D-A_{i}\right)\right)$.

(ii) Suppose that there do exist elements $x$ and $y$ of $m$ such that $\pi^{*}(x)$ and $\pi^{*}(y)$ restrict to linearly independent (over $\mathbf{C})$ elements of $\Gamma\left(A_{i}, \mathcal{O}(-D) / \mathcal{O}\left(-D-A_{i}\right)\right)$. Then (3.1) holds for this $A_{i}$.

Proof. (i) Suppose that $A_{i} \cdot D<0$. Then $\mathcal{O}(-D) / \mathcal{O}\left(-D-A_{i}\right)$ is the sheaf of germs of sections of a line bundle $L$ over $A_{i}$ of positive chern class. Choose $x$, an element of $\mathfrak{m}$, such that $\pi^{*}(x)$ does not vanish identically on $A_{i} \cdot \pi^{*}(x)$ has a zero, say at $q$, as a section of $L$. In the absence of embedded points for $\pi^{*}(\mathfrak{m})$, there must be a second element $y$ of $m$ such that $\pi^{*}(y)$ is nonzero at $q$ as a section of $L$. Then $x$ and $y$ are the desired elements of $m$.

(ii) Let $F(x, y, z)=0$ be a defining equation for $V$ near $p$. Then

$$
\omega=\frac{d x \wedge d y}{F_{z}}=\frac{d y \wedge d z}{F_{x}}=\frac{d z \wedge d x}{F_{y}}
$$

is a nowhere zero meromorphic 2-form with $p$ as its only pole. Then the divisor $\left(\pi^{*}(\omega)\right)$ equals $K$. We shall prove Proposition 3.2 by doing a calculation for $\pi^{*}(\omega)$ near $A_{i}$ : Since $\pi^{*}(x)$ and $\pi^{*}(y)$ project to linearly independent sections of $L$, 
$d x \wedge d y$ vanishes to order exactly $2 d_{i}-1$ on $A_{i}$. Since $V$ has multiplicity $\nu$ at $p$, $F(x, y, z)$ has a zero of order $\nu$ at $p$. Hence $F_{z}$ has a zero of order at least $\nu-1$ at $p$. Then $\pi^{*}\left(F_{z}\right)$ has a zero or order at least $(\nu-1) d_{i}$ on $A_{i}$. Hence $k_{i} \leqslant 2 d_{i}-1-$ $(\nu-1) d_{i}$. This is (3.1).

Suppose that $\pi:(M, A) \rightarrow(V, p)$ and $\pi^{\prime}:\left(M^{\prime}, A^{\prime}\right) \rightarrow(V, p)$ are two resolutions of $(V, p)$ such that $\pi^{\prime}=\lambda \circ \pi$ for a suitable composition of quadratic transformations $\lambda$. Then

$$
K^{\prime}=\lambda^{-1}(K)+J
$$

where $\lambda^{-1}(K)$ is the total transform of $K$ and $J$ is the divisor of the Jacobian of $\lambda$. In particular, if $A_{i}$ is an irreducible component of $A$, let $A_{i}^{\prime}=\lambda^{*}\left(A_{i}\right)$, the proper transform of $A_{i}$. Then $k_{i}^{\prime}=k_{i}$. Also, in the special case that $\lambda$ is the blowup at a single point $q, J=\lambda^{-1}(q)$.

Now consider Theorem 3.1 for the case $\nu=2$, double points. Recall [10, p. 428]. Since $d_{i} \geqslant 1$, to prove (3.1) it suffices to show that $k_{i} \leqslant 0$. If $\pi$ is the minimal resolution, in fact $k_{i} \leqslant 0$ by Proposition 2.1. In any event, start by taking $\pi$ as the minimal resolution. If $D \cdot D=-2$ when $\pi$ is the minimal resolution, then $\pi$ must be the minimal resolution in Theorem 3.1. With $\nu=2, D \cdot D=-1$ is the only other possibility when $\pi$ is the minimal resolution. Then in fact $D=Z$, the fundamental cycle [1] and $\pi^{*}(\mathfrak{m})$ has an embedded point $q$. The only other candidate $\pi^{\prime}$ for $\pi$ in Theorem 3.1 is to blow up, via $\lambda$, the minimal resolution at $q$. By Proposition 3.2, (3.1) holds for $\lambda^{-1}(q)$. (3.2) completes the proof of Proposition 3.3 below for the other irreducible components of $A^{\prime}$.

Proposition 3.3. Theorem 3.1 is true when $\nu=2$.

Proposition 3.4. Let $\pi:(M, A) \rightarrow(V, p)$ be as in Theorem 3.1. Consider an $A_{i}$ in A. Suppose that $\pi^{*}(\mathrm{~m})$ has an embedded point at a point $q$ in $A_{i}$ such that $q$ is a smooth point of $A$. Then $-k_{i} \geqslant(\nu-1)+(\nu-3) d_{i}$.

Proof. Consider the situation of (3.2) where $\lambda$ consists of one blowup at $q$. Let $A_{l}=\lambda^{-1}(q)$. Then

$$
D^{\prime}=\lambda^{-1}(D)+c A_{l}, \quad c \geqslant 1 .
$$

From (3.2) and (3.3), we see that for each $A_{i}$ we may associate a $k_{i}$ and $d_{i}$ which remain unchanged under taking proper transforms.

Drop the subscript $i$. Successively blow up at $q$ and subsequent embedded points for as long as possible, naming the new curves (and their proper transforms) by the subscripts $1,2, \ldots, t, \ldots$ We claim that, by induction on $t$, for each $t \geqslant 1$, there exist positive integers $a_{t}$ and $b_{t}$ such that

$$
k_{t}=a_{t} k+b_{t}, \quad d_{t} \geqslant a_{t} d+b_{t}, \quad b_{t} \geqslant a_{t} .
$$

Since $q$ is a smooth point of $A$, for $t=1$, via (3.2) and (3.3), (3.4) holds with $a_{t}=b_{t}=1$. Each subsequent blowup occurs either at a smooth point of the exceptional set, in which case (3.4) follows by induction as for $t=1$ via (3.2) and (3.3), or at the transverse intersection of two curves, call them $A_{r}$ and $A_{s} \cdot r=0$, 
corresponding to $r=i$, is possible here. $a_{0}=1$ and $b_{0}=0$. Then

$$
\begin{aligned}
& k_{t}=k_{r}+k_{s}+1=\left(a_{r}+a_{s}\right) k+\left(b_{r}+b_{s}+1\right), \\
& d_{t} \geqslant d_{r}+d_{s}+1
\end{aligned}
$$

and (3.4) follows.

Let $A$, be the result of the last blowup. Then $l \leqslant \nu-1$. By Proposition 3.2, $-k_{l} \geqslant 1+(\nu-3) d_{l}$. By Proposition 3.3, we may take $\nu \geqslant 3$. Then

$$
\begin{aligned}
& -a_{l} k-b_{l} \geqslant 1+(\nu-3)\left(a_{l} d+b_{l}\right), \\
& -k \geqslant\left(1 / a_{l}\right)+(\nu-2)+(\nu-3) d .
\end{aligned}
$$

Since $a_{l}$ is positive, this proves Proposition 3.4.

Lemma 3.5. Let $\pi:(M, A) \rightarrow(V, p)$ be as in Theorem 3.1. If $\nu \geqslant 3$, then $-k_{i} \geqslant 1$ for all $i$.

Proof. Start with $\pi$ as the minimal resolution. Then $-k_{i} \geqslant 1$ by Proposition 2.1 . Now blow up $M$ successively at embedded points which occur at singularities of $A$, i.e. where $A$ has multiplicity greater than 1 . Then, by (3.2), also $-k_{i} \geqslant 1$ for any such new exceptional curves. Eventually we reach a resolution where each embedded point satisfies the hypotheses of Proposition 3.4. At most $(\nu-1)$ additional blowups are needed to reach the minimal resolution $\pi^{\prime}$ with $\pi^{\prime *}(\mathfrak{m})$ locally principal. By Proposition 3.4 and (3.2), for $\nu \geqslant 4$, each blowup yields a new $A_{i}$ with $-k_{i} \geqslant 1$, proving Lemma 3.5 for $\nu \geqslant 4$. For $\nu=3$, each blowup except possibly the last yields a new $A_{i}$ with $-k_{i} \geqslant 1$. The last $A_{l}$ satisfies the conditions of Proposition 3.2. Hence also $-k_{l} \geqslant 1$.

Observe that Lemma 3.5 yields Theorem 3.1 for $\nu=3$.

It now suffices to prove Theorem 3.1 in the case that $\nu \geqslant 4$ and $\pi^{*}(\mathrm{~m})$ is locally principal. We claim, in analogy to the existence of the fundamental cycle $Z$, that $(\nu-3) D$ is the minimal cycle $C=\sum c_{i} A_{i}$ such that

$$
\begin{array}{ll}
C \geqslant 0, & \\
c_{i} \geqslant(\nu-3) d_{i} & \text { for } A_{i} \cdot D<0, \\
A_{i} \cdot C \leqslant 0 & \text { for } A_{i} \cdot D=0 .
\end{array}
$$

Since the (component-wise) minimum of two cycles which satisfy (3.5) also satisfies (3.5), a unique minimal $C$ does exist. $(\nu-3) D$ does satisfy (3.5). Write

$$
(\nu-3) D=C+P, \quad P \geqslant 0 .
$$

Let $P=\sum p_{i} A_{i}$. If $A_{j} \cdot D<0$, then $p_{j}=0$ and $A_{j} \cdot P \geqslant 0$. If $A_{j} \cdot D=0$, then

$$
0=A_{j} \cdot C+A_{j} \cdot P \leqslant A_{j} \cdot P .
$$

Hence $P \cdot P \geqslant 0$ and by negative-definiteness, $P=0$.

By Lemma $3.5,-K \geqslant 0$. By Proposition $3.2,-k_{j} \geqslant(\nu-3) d_{j}$ for $A_{j} \cdot D<0$. By the adjunction formula and since $A_{j} \cdot D<0$ if $A_{j}$ is exceptional of the first kind, $A_{j} \cdot(-K) \leqslant 0$ if $A_{j} \cdot D=0$. That is, $-K$ satisfies (3.5). Then $-K \geqslant(\nu-3) D$. Since in fact, $-k_{i} \geqslant 1+(\nu-3) d_{i}$ for $A_{i} \cdot D<0$ and $-K \cdot A_{i} \leqslant 0$ for $A_{i} \cdot D=0$, a computation sequence argument as in [5] and the connectedness of $A$ yields (3.1) for all $i$. This completes the proof of Theorem 3.1. 
COROLlaRY 3.6. Let $(V, p)$ be an isolated two-dimensional hypersurface singularity with multiplicity $\nu, \nu \geqslant 4$. Let $K$ be the canonical divisor on the minimal resolution of $(V, p)$. Then

$$
-K \cdot K \geqslant 2+\nu(\nu-2)(\nu-4)
$$

Proof. Suppose a resolution $\pi^{\prime}$ of $(V, p)$ is obtained from some other resolution $\pi^{\prime \prime}$ by a single blowup. Let $K^{\prime}$ and $K^{\prime \prime}$ denote the canonical divisors for $\pi^{\prime}$ and $\pi^{\prime \prime}$ respectively. By $(3.2),-K^{\prime} \cdot K^{\prime}=-K^{\prime \prime} \cdot K^{\prime \prime}+1$. Now let $\pi$ denote the minimal resolution and let $\pi^{\prime}$ be the minimal resolution with $\pi^{\prime *}(\mathfrak{m})$ locally principal. There are at most $(\nu-1)$ blowups between $\pi$ and $\pi^{\prime}$. Thus

$$
-K^{\prime} \cdot K^{\prime} \leqslant-K \cdot K+(\nu-1) \text {. }
$$

By Theorem $3.1,-K^{\prime}>(\nu-3) D^{\prime}$. Since $A_{i} \cdot D^{\prime} \leqslant 0$ for all $i$,

$$
K^{\prime} \cdot K^{\prime}<(\nu-3)^{2} D^{\prime} \cdot D^{\prime}=\nu(\nu-3)^{2} .
$$

(3.7) and (3.6) yield the corollary.

We have the following necessary conditions on a weighted dual graph $\Gamma$ to come from a hypersurface singularity.

COROLlary 3.7. Let $\Gamma$ be the weighted dual graph of the minimal resolution of an isolated hypersurface singularity. Then there is a cycle $K=\sum k_{i} A_{i}$ on $\Gamma$, with integer coefficients, which satisfies the adjunction formula. Let $Z=\sum z_{i} A_{i}$ be the fundamental cycle. Then, for all $i,-k_{i} \geqslant 1+(-Z \cdot Z-3) z_{i}$.

Proof. Let $D$ be the maximal ideal cycle on $A$. Then $D \geqslant Z$. Moreover, by [1], the multiplicity $\nu$ satisfies

$$
\nu \geqslant-Z \cdot Z \text {. }
$$

Inequality (3.8) and Corollary 3.6 prove the corollary for $-Z \cdot Z \geqslant 3$. For $-Z \cdot Z \leqslant$ 2 , the corollary follows from Proposition 2.1 , but is essentially vacuous.

IV. Examples. The general results of §III do not always yield results which are as complete as analyses which use techniques such as in [8 and 5]. However, §III does allow a more rapid, if less complete, calculation in many cases.

EXAMPLE 4.1. Recall the simply elliptic singularities [8], i.e. singularities $(V, p)$ which have a minimal resolution $(M, A)$ with $A$ a smooth elliptic curve. $(V, p)$ is Gorenstein [5]. It is known [8] that $(V, p)$ is a hypersurface precisely for $1 \leqslant-A \cdot A$ $\leqslant 3$. See also [5]. Let us apply Corollary 3.7. For all $A \cdot A, K=-A$ and $Z=A$ on the minimal resolution. Then $k_{i}=-1$ and $z_{i}=1$. So a necessary condition for a simply elliptic singularity to be a hypersurface is $1 \geqslant 1+(-A \cdot A-3)$, or $-A \cdot A \leqslant$ 3. The condition of Corollary 3.7 is sharp in this family.

EXAmple 4.2. Consider the weighted dual graph $\Gamma$ of (4.1). The central curve has genus 1 , while the arms have genus 0 .

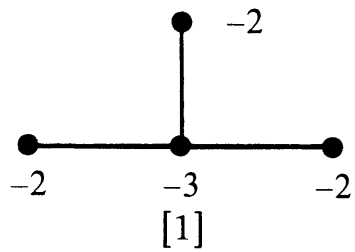


A hypersurface representative, of multiplicity 3 , may be given for $\Gamma$ by $\left\{x^{3}+y^{3}+\right.$ $\left.z^{9}=0\right\}$.

Let $(V, p)$ be an arbitrary isolated hypersurface singularity with $\Gamma$ as the weighted dual graph of its minimal resolution. Number the vertices of $\Gamma$ as in (4.2). Let $\nu$ denote the multiplicity of $(V, p)$.

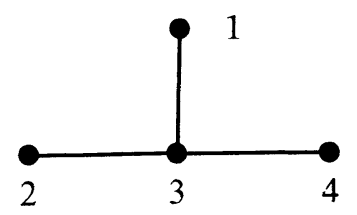

Then $Z=A_{1}+A_{2}+A_{3}+A_{4} \cdot Z \cdot Z=-3$, so $\nu \geqslant 3 . K=-A_{1}-A_{2}-2 A_{3}-A_{4}$. Let $D$ be the maximal ideal cycle. Then $D \geqslant Z$. From Theorem 3.1, with $i=1,2$, or $4, \nu=3$. Then also, $D=Z$ and there are no embedded points for $\pi^{*}(\mathrm{~m})$.

By way of contrast, consider a (non-Gorenstein) [5] singularity given by a "generic" choice of the complex structure on $(M, A)$ with weighted dual graph $\Gamma$ of (4.1). Then the line bundle $L$ on $A_{3}$ such that $\mathcal{O}(L)=\mathcal{O}(-Z) / \mathcal{O}\left(-Z-A_{3}\right)$, of chern class $A_{3} \cdot Z=0$, is not the trivial bundle and so lacks sections. Then $D>Z$ and $\nu \geqslant 4$.

EXAMPLE 4.3. Even with the Milnor number $\mu$ [6], the weighted dual graph $\Gamma$ cannot always determine the multiplicity of an isolated hypersurface $(V, p)$. Consider $\Gamma$ of (4.3). As in (4.1), genera equal to 0 are omitted from the labeling.

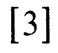

Here are some weighted-homogeneous representatives for $\Gamma$ with their multiplicities, Milnor numbers, and characteristic polynomials $\Delta(t)[6]$.

$$
\begin{array}{cccc}
\text { Equation } & \nu & \mu & \Delta(t) \\
z^{2}+x^{7}+y^{42}=0 & 2 & 246 & (t-1)^{-1}\left(t^{2}-1\right)\left(t^{14}-1\right)^{-1}\left(t^{42}-1\right)^{6} \\
z^{2}+y\left(x^{12}+y^{18}\right)=0 & 2 & 210 & (t-1)^{-1}\left(t^{2}-1\right)\left(t^{19}-1\right)\left(t^{38}-1\right)^{5} \\
z^{3}+x^{4}+y^{36}=0 & 3 & 210 & (t-1)^{-1}\left(t^{3}-1\right)\left(t^{4}-1\right)\left(t^{12}-1\right)^{-1}\left(t^{36}-1\right)^{6}
\end{array}
$$

The author knows of no isolated hypersurface $(V, p)$ having (4.3) as the weighted dual of its minimal resolution which does not lie in a $\{\mu=$ constant $\}$ family which contains one of the three singularities in (4.4). III provides some limited conditions on such a $(V, p)$, as follows. Number the vertices as in (4.5).

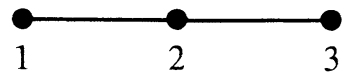

Then $Z=A_{1}+A_{2}+A_{3}$ and $K=-15 A_{1}-10 A_{2}-5 A_{3} . D \geqslant Z$. Then Theorem 3.1 with $i=3$ yields $\nu \leqslant 7$. By considering the various possibilities for $D$, we shall show further that $\nu \leqslant 6$. Recall [1] that for all $A_{i}, A_{i} \cdot D \leqslant 0$.

Suppose that $D=Z$. Since $Z \cdot Z=-1$ and a multiplicity of 1 is not possible for $(V, p), \pi^{*}(\mathfrak{m})$ has an embedded point $q . q$ necessarily satisfies the hypotheses of Proposition 3.4 with $i=3$. The integer $\nu$ satisfies $\nu \leqslant 4$. 
For $D>Z$, necessarily $D \geqslant Z_{1}$, with $Z_{1}=2 A_{1}+2 A_{2}+A_{3}$. Consider the case $D=Z_{1}$. If there are no embedded points, then $\nu=-D \cdot D=2$. Any embedded point must occur at a point $q$ which satisfies the hypotheses for Proposition 3.4 with $i=2$. Then $\nu \leqslant 5$.

If $D>Z_{1}$, then $D \geqslant 2 Z$ or $D=Z_{2}$, with $Z_{2}=3 A_{1}+2 A_{2}+A_{3}$. For $D \geqslant 2 Z$, Theorem 3.1 with $i=3$ yields $\nu \leqslant 5$. Consider now the final case: $D=Z_{2}$. If there are no embedded points, then $\nu=-D \cdot D=-3$. Any embedded point must occur at a point $q$ which satisfies the hypotheses for Proposition 3.4 with $i=1$. Then $\nu \leqslant 6$.

AdDEndum. Using a result of Y. Koyama, M. Tomari has proved the following stronger consequence of the hypotheses of Corollary 3.7: If $\nu$ is even, then $-K \cdot K \geqslant$ $(\nu-2)^{2} \nu$. If $\nu$ is odd, then $-K \cdot K \geqslant \nu(\nu-1)(\nu-3)$.

\section{REFERENCES}

1. M. Artin, On isolated rational singularities of surfaces, Amer. J. Math. 88 (1966), 129-136.

2. H. Bass, On the ubiquity of Gorenstein surfaces, Math. Z. 82 (1963), 129-136.

3. H. Grauert, Über Modifikationen und exzeptionelle analyticshe Mengen, Math. Ann. 146 (1962), 331-368.

4. H. Grauert and O. Riemenschneider, Verschwindungssatze für analytische Kohomologie gruppen auf Komplexen Raumen, Invent. Math. 11 (1970), 263-292.

5. H. Laufer, On minimally elliptic singularities, Amer. J. Math. 99 (1977), 1257-1295.

6. J. Milnor, Singular points of complex hypersurfaces, Ann. Math. Studies, no. 61, Princeton Univ. Press, Princeton, N. J., 1968.

7. W. Netamann, A calculus for plumbing applied to the topology of complex surface singularities and degenerating complex curves, Trans. Amer. Math. Soc. 268 (1981), 299-344.

8. K. Saito, Einfach-elliptische Singularitaten, Invent. Math. 23 (1974), 289-325.

9. J. P. Serre, Groupes algebriques et corps de classes, Act. Sci. Indust. 1264, Hermann, Paris, 1959.

10. P. Wagreich, Elliptic singularities of surfaces, Amer. J. Math. 92 (1970), 419-454.

11. S. S.-T. Yau, Normal singularities of surfaces, Proc. Sympos. Pure Math., Vol. 32, Amer. Math. Soc., Providence, R. I., 1978, pp. 195-198.

12. O. Zariski, Some open questions in the theory of singularities, Bull. Amer. Math. Soc. 77 (1971), $481-491$.

Department of Mathematics, State University of New York at Stony Brook, Long Island, NEW YORK 11794 